\title{
Achieving Convergence through Deployment of IPTV in Cellular Network
}

\author{
Nazmus Sakib \\ Lecturer, Ahsanullah University of \\ Science \& Technology
}

\author{
Hasib-Bin-Shakur \\ Lecturer, Stamford University of \\ Bangladesh
}

\author{
Tanveer M. Haque \\ Senior Executive, Grameen Phone \\ Limited, Bangladesh
}

\begin{abstract}
Present IPTV system fails to establish QoS due to packet loss, server congestion and limited bandwidth. To overcome these drawbacks an implementation of IPTV is presented here using MPLS in 3GPP LTE release 8 networks. This implementation will provide multicast capability and FEC along with retransmission to minimize packet loss. Deploying IPTV in LTE makes LTE capable of transmitting voice, data and audio/video which will ensure convergence.
\end{abstract}

\section{Keywords}

3GPP LTE Release 8, IPTV, MPLS, MPEG-4 Part 10, and FEC.

\section{INTRODUCTION}

Internet Protocol Television (IPTV) provides multimedia services such as television, video, audio, text, graphics, and data through IP based networks. It also ensures the required Quality of Service (QoS), experience, security, interactivity, and reliability [1]. IPTV offers carrier flexibility and adds value in the form of additional services that can be offered to customers, which improves the carrier's profitability and competitive edge. IPTV provides "triple play" [2] of services: voice, data, and video.

In the proposed architecture, IPTV has been implemented using Multi-Protocol Label Switching (MPLS) and Long Term Evolution (LTE) Release 8 network to obtain higher QoS guarantee and user connectivity. Moreover, implementing IPTV in LTE Release 8 network means both voice and data along with audio and video can be transmitted over the same connection. For video encoding, Moving Picture Experts Group 4 (MPEG-4) Part 10 is proposed in this architecture as it minimizes the bandwidth requirements and guarantees bandwidth efficiency. Forward Error Correction (FEC) along with retransmission based retrieval schemes is proposed to ensure the robustness of the architecture in case of data loss.

Third Generation Partnership Project (3GPP) defines some requirements for LTE which includes reduced cost per bit, increased service provisioning - more services at lower cost with a better user experience, flexibility of use of existing and new frequency bands, simplified architecture, and reasonable terminal power consumption. In terms of actual figures, targets for LTE included absolute peak rates of download 300 $\mathrm{Mb} / \mathrm{s}$ (4 layers) and upload $75 \mathrm{Mb} / \mathrm{s}$ (1 layer, 64-QAM) within $20 \mathrm{MHz}$ bandwidth [3], support at least 200 active users in every $5 \mathrm{MHz}$ cells, and sub-10ms latency for small IP packets [4].

MPLS defines a mechanism for faster packet forwarding in network routers. The flexibility of MPLS has led to it becoming the default way for modern networks to achieve QoS, next generation VPN services, and optical signaling.
MPEG-4 is an ISO/IEC standard developed by Moving Picture Experts Group (MPEG). It is a collection of compression methods for audio and visual (AV) digital data. It is a compact solution model which refers to the utility features of MPEG-1, MPEG-2, and other related standards to support different types of interactivity.

FEC allows the receiver to detect and correct errors (within some bound) without the need to ask the sender for additional data. The advantage of FEC is that it eliminates the need of back-channel [5].

\section{PRESENT IPTV}

IPTV maintains a process of preparing and distributing television signals in which content streams are acquired first then demodulated and decrypted, if necessary, and finally reencoded digitally for transporting with additional compression and advanced encryption over the IP based network. IPTV signals, or streams, are distributed on IP based network and viewed on an IPTV enabled viewing device, usually a set-top box. Different service providers of IPTV use their own technology for providing IPTV service to the users. But the key steps that are involved in the existing satellite based transmission of IPTV include: acquiring the programming, encoding the streams, IPTV distribution network, middleware, IPTV viewing device.

Programming sources are simply the channels that provide programming for broadcasting. The provider does not create original programming itself; it pays other companies for the right to broadcast their content via satellite. The broadcast center converts all of this programming into a high-quality, uncompressed digital stream.

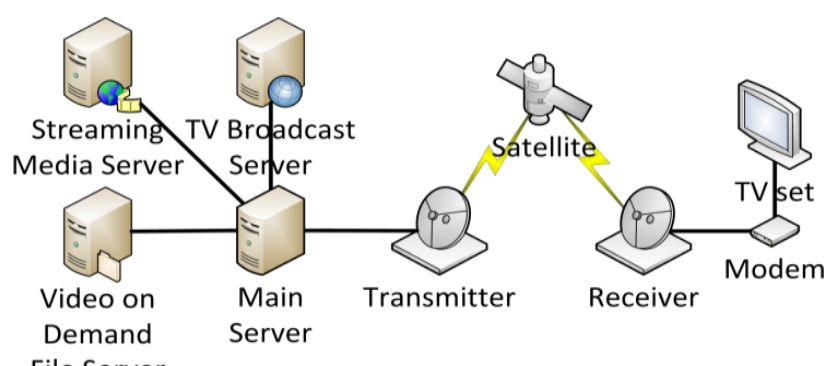

Fig. 1: Present IPTV

In present IPTV network, main server is the broadcast center that also acts as the central hub of the system (Fig. 1). The broadcast center receives signals from various programming sources and beams a broadcast signal to satellites in geosynchronous orbit. At the broadcast center, the highquality digital stream of video goes through an MPEG encoder, which converts the programming to MPEG-4 video of the correct size and format for the satellite receiver in a viewer's house. Once the signal is compressed and encrypted, the broadcast center beams it directly to one of its satellites. 
The satellite picks up the signal with an onboard dish and then amplifies the signal. It uses another dish to beam the signal back to Earth, where viewers can pick it up [6].

When the signal reaches the viewer's house, it is captured by the viewer's satellite dish. A satellite dish is just a special antenna designed to focus on a specific broadcast source. The viewer's dish picks up the signal from the satellite (or multiple satellites in the same part of the sky) and passes it on to the receiver in the viewer's house. The end component in the entire satellite TV system is the receiver. It processes the signal and passes it on to a standard TV [6].

Deploying IPTV in satellite based network arises some problems.

i. Interactivity

Satellite network is unable to provide user interactive services because only receiver is used. Moreover, using transceiver is very much costly

ii. Signal latency

The signal delay can be as much as 250 milliseconds to 900 milliseconds (one way), which makes this service unusable for applications requiring the realtime user input and can be irritating with interactive applications, such as VoIP, video conferencing, or other person to person communication.

\section{iii. Weather Effect}

Satellite communications are affected by moisture, rain and snow in the signal path between end users or ground stations and the satellite being utilized [7].

\section{iv. Line of sight}

Typically an absolutely clear line of sight between the dish and the satellite is required for the system to work.

Moreover, when servers are not provisioned to support the maximum number of expected users, then server congestion can occur. This generally leads to pauses in video playback as the playout buffer level is too low [8] [9].

\section{PROPOSED ARCHITECTURE}

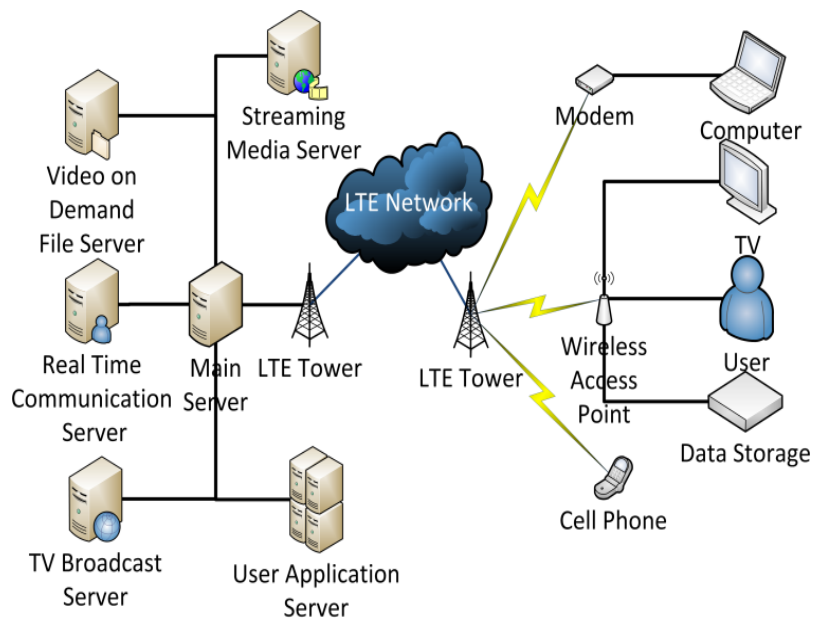

Fig. 2: Proposed Architecture
Proposed architecture is an implementation of IPTV using the cellular network (Fig. 2), whereas the present IPTV is mostly satellite based. As most of the IPTV service providers are telecom companies, therefore, a solution to provide IPTV using their existing cellular network will help them to get rid of setting up a new satellite network just for IPTV. Moreover, if the service can be provided using a cellular network; vast array of user interactivities can also be provided.

3GPP LTE network is the backbone of the proposed architecture. LTE is designed to support peak data rates of $300 \mathrm{Mbps}$ in downlink and $75 \mathrm{Mbps}$ in uplink within a $20 \mathrm{MHz}$ bandwidth [10]. Improvements in architecture and signaling reduce round-trip latency [11] [3]. To suit as many frequency band allocation arrangements as possible, both paired (FDD) and unpaired (TDD) band operation is supported [12]. 3GPP's core network is optimized for packet mode and in particular for IP-Multimedia Subsystem (IMS) which supports all access technologies [3]. Multiple Input Multiple Output (MIMO) antenna technology enables 10 times as many users per cell as 3GPP's original W-CDMA radio access technology [13].

In case of 3GPP LTE, voice is transmitted in packet switched network. LTE is an IP based network that provides end-to-end IP connection from the mobile equipment to the core network. VoIP is used for providing voice support in LTE.

3GPP LTE network can maintain the path to provide basic services of IPTV as well as the cell phone services to the end user with mobility.

IPTV concept holds some basic services to viewers. There are mainly three types of services that IPTV provides:

i. TV service that includes broadcasting of live streaming video, audio and also stored TV program.

ii. Video on demand service, which includes access, to movies and other video content as per user requirement

iii. Subscription based service means user interactive service in general.

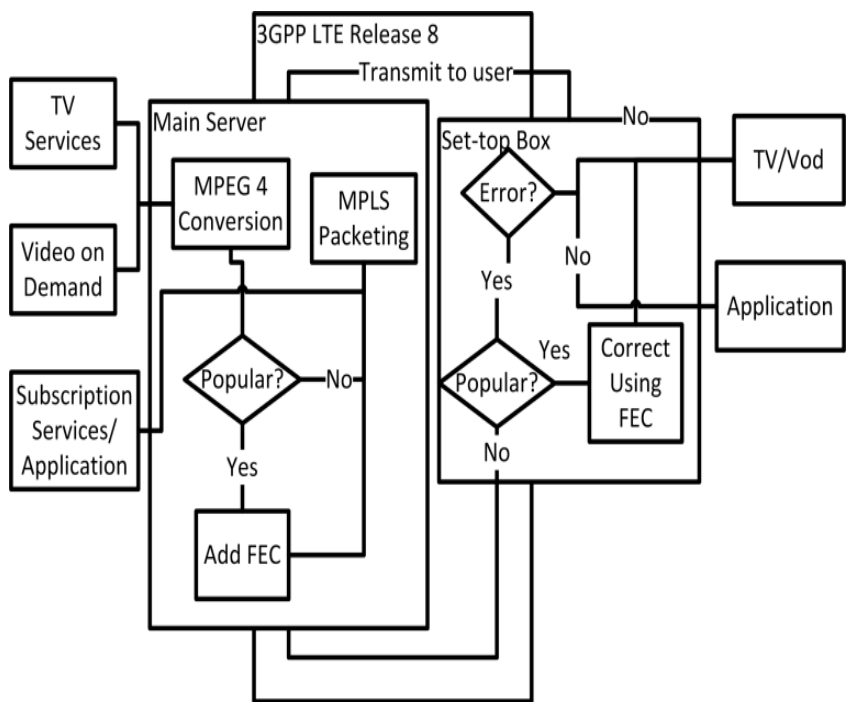

Fig. 3 Block Diagram 
The block diagram of the proposed architecture has three types of services which are stored in the designated servers (Fig. 3). Audio visual elements are stored in MPEG-4 part 10 format. The reason of using MPEG-4 part 10 is that it provides DVD quality video and uses lower bit rate so that it is feasible to transmit digitized video streams in the internet where bandwidth is more critical, and hard to guarantee. Motion Joint Photographic Experts Group (MJPEG's) bandwidth requirement is a little bit more than MPEG-4 while its resolution and frame rate are much worse. MPEG-2 can provide DVD-like video, just like MPEG-4. However, MPEG-2's bandwidth requirement is so high that it is not possible to use it in an IP network [14] [15].

When a user requests for a TV channel or any video on demand content then the contents are forwarded to the main server from their designated servers. MPEG-4 video traffic must be split into many packets to be transmitted in the MPLS network (Fig. 5).

In order to adapt with the architecture of MPLS, one packet is not considered as a unit of transmission, instead one Video Object Plane (VOP) is considered as a unit of transmission. Each VOP consists of many MPEG-4 packets (Fig. 4). Moreover, the packets of one VOP have the same Forwarding Equivalence Class (FEC) using MPLS. Hence, the packets belonging to one VOP traverse the same LSP which means they are transmitted along the same path. Whenever, the network resource is not enough (unavailability of high bandwidth) or congestion occurs at the router, then all packets of one VOP that has low priority is discarded instead of discarding some packets randomly to reserve bandwidth for other VOPs of high priority [16].
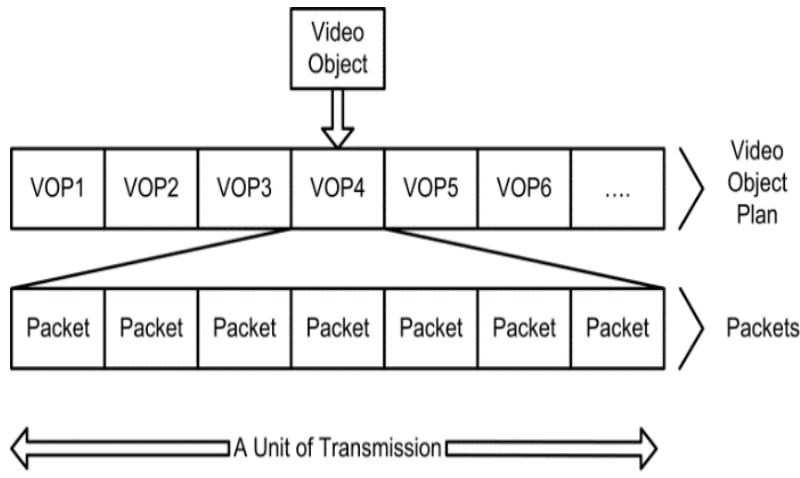

Fig. 4: Packetized MPEG-4 video traffic [16]

The packets are then passed to the server for checking popularity of the program. Popularity checking is necessary because based on the popularity it is determined whether the service is going to use FEC or retransmission based recovery schemes [17].

To determine the popularity of a channel, one method that can be used is to count the number of recipients of a multicast stream. Alternatively it can be determined by the number of retransmission requests over a given period of time. Another way of determining the popularity is to predict the popularity on a priority basis, like, determining the popularity of a weekly TV-show. This pre-computation substantially reduces the computational overhead. Another scenario may arise that a channel may be popular in one segment of the network and not popular in another segment. Then such channels are distributed using FEC in popular regions, not in the unpopular regions. This type of popularity measure is called local popularity checking methods [17].

Error in popular channel is handled through the use of FEC. FEC produces a fixed overhead for popular channels, which is independent of the number of users. This means number of errors to correct scales with the popularity.

Error in less popular channel is handled by retransmission. As there are fewer users of less popular channels at a given time the overhead experienced by retransmission is smaller than the overhead associated with FEC for the unpopular channels.

The data for TV and video on demand service is sent to MPLS-Networks [16] [18] after checking popularity where the interactive subscription services are directly send to the MPLS-Networks. MPLS works by tagging packets with an identifier (a label) to distinguish the Label Switched Paths (LSPs). When a packet is received, the router uses this label (and sometimes also the link over which it was received) to identify the LSP. It then looks up the LSP in its own forwarding table to determine the best link over which to forward the packet and the label to use on this next hop. A different label is used for each hop, and it is chosen by the router or switch performing the forwarding operation. This allows the use of extremely fast and simple forwarding engines, as the router can select the label to minimize processing.

Here, MPLS is used because, in a pure IP network, the shortest path to a destination is chosen even when it becomes more congested. Meanwhile, in an IP network with MPLS Traffic Engineering CSPF routing, constraints such as the RSVP bandwidth of the traversed links can also be considered, such that the shortest path with available bandwidth will be chosen. Moreover, In the event of a network element failure, MPLS local protection meets the requirements of real-time applications with recovery times comparable to those of SONET rings of less than 50ms [19]. Multicast is also possible in MPLS-networks which is very much necessary for IPTV.

The packets are then sent to the service provider set-top-boxes over LTE network. LTE has introduced a number of new technologies which will enable IPTV to operate more efficiently [3] such as Orthogonal Frequency Division Multiplex which provides high data bandwidths along with a high degree of resilience to reflections and interference [3], Multiple-input and multiple-output which improves communication performance and increases the throughput [20], and System Architecture Evolution [21]. 


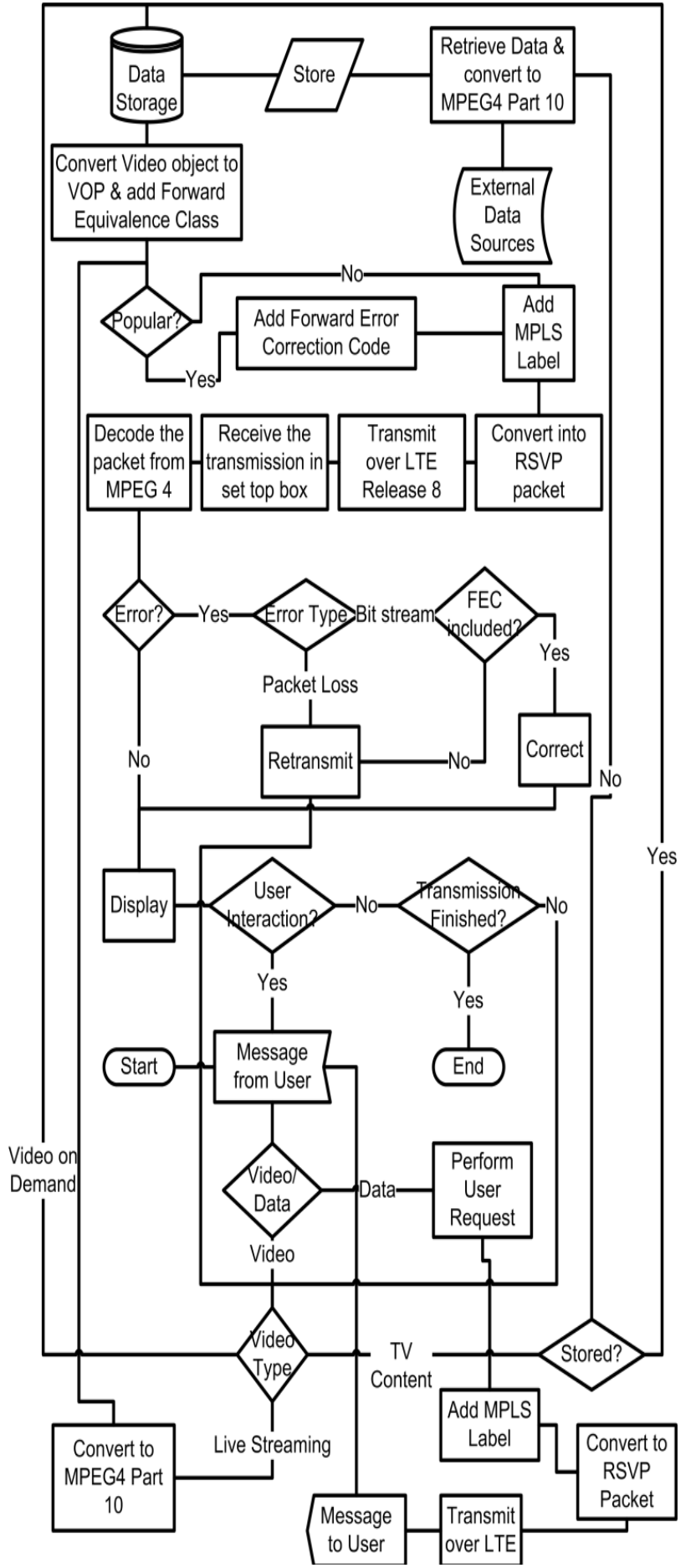

Fig. 5: Flow Diagram

MPLS-capable network elements can address LTE all-IP routing requirements by delivering Layer 3-based functionality.

In the receiver side set top boxes if there are errors for the popular channels then they are fixed with FEC and for nonpopular channels packets are requested from the server. FEC decoder consists of mainly three components: control channel decoder, data channel decoder, and hybrid automatic repeat query (HARQ) combining. To guarantee the reliable data transmission over wireless channels, 3GPP LTE employ hybrid ARQ that incorporates forward error correction code with the retransmission scheme to ensure reliable transmission of data packets.

In this proposed architecture user can subscribe for various services. If customer premises equipment receives any interaction from the user, it sends the data to the server as normal datagram packets. After satisfying the requests from the user the server sends the output to him. This unicast system ensures user interactivity.

\section{OVERCOMING DIFFICULTIES}

In LTE full duplex communication is possible, so user interactive service can be provided. In satellite network signal latency is around $250 \mathrm{~ms}$, but using LTE we can reduce the latency below $10 \mathrm{~ms}$ [4]. Moisture, rain, and snow cannot affect the signal strength of cellular network. LTE is a nonline of sight network. Hence, it overcomes the problem of obstacle in the signal path.

\section{ACHIEVING CONVERGENCE}

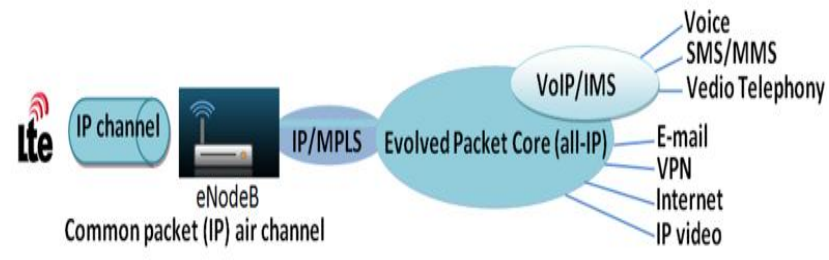

Fig. 6 Achieving Convergence

In case of LTE based network, it uses the idea of transmitting voice and IP data packets along with audio visual elements to the intended receiver to achieve convergence (Fig. 6). All types of packets are transmitted through the IP/MPLS architecture to the Evolved Packet Core (EPC) which is a converged core networking architecture for transmitting all types of packets voice, video telephony, SMS, IP video, email, VPN to their intended receiver.

\section{CONCLUSION}

Here, it has been shown that IPTV is possible to implement in the existing mobile network to provide the facility of user interactivity and convergence. This proposed architecture describes the functionality and reliability of IPTV with a vast technology of telecommunication that is 3GPP LTE release 8 .

\section{REFERENCES}

[1] Hwang, "Content and Service Protection for IPTV," IEEE TRANSACTIONS ON BROADCASTING, vol. 55 no. 2, pp. 425-436, JUNE 2009.

[2] Nemeth, "Brave new world of telecom," in 9th International Conference on Telecommunications, Zagreb, 2007, pp. 339-342.

[3] Berkmann, Carbonelli, Dietrich, Drewes, and Xu , "On 3G LTE Terminal Implementation - Standard, Algorithms, Complexities and Challenges," in Proceedings of International Wireless Communications and Mobile Computing Conference, Crete Island, March 2008, pp. 970-975. 
[4] Sel'en and Asplund, "3G LTE Simulations Using Measured MIMO Channels," in IEEE Global Communications Conference, New Orleans, 2008.

[5] Clark, J. George, and C. J. Bibb, Error-Correction Coding for Digital Communications. New York, United States of America: Plenum Press, 1981.

[6] T. Harris and Nice. (2010, May) How Stuff Works. [Online]. Available: http://electronics.howstuffworks.com/satellitetv.htm/printable

[7] T. I. Ed, Satellite Communications: System and Its Design Technology, 2th ed., T. I. Ed, Ed. Tokyo, 1018460, Japan: IOS Press, 2000.

[8] Telchemy Incorporated, "Managing IPTV Performance," Telchemy Incorporated, February 2008. [Online]. Available: www.telchemy.com/appnotes/Managing_IPTV_Perfor mance.pdf

[9] M. Atenas, Canovas, Garcia, and Lloret, "IPTV Transcoding to Avoid Network Congestion," in Sixth International Conference on Networking and Services, Cancun, Mexico , 2010, pp. 36-41.

[10] D. M. Sacristán et al., "On the Way towards FourthGeneration Mobile: 3GPP LTE and LTE-Advanced," EURASIP Journal on Wireless Communications and Networking, vol. 2009 (2009), no. 354089, p. 10, June 2009.

[11] Shi, Z. Ji, Z. Gao , and L. Huang, "Layered Security Approach in LTE and Simulation," Xiamen University, Xiamen, Fujian,

[12] Y. Wang, S. Frattasi, T. B. Sørensen, and P. E. Mogensen, "Network Time-Synchronization in TDD Based LTE-Advanced Systems," in 69th Vehicular Technology Conference, Barcelona, 2009.

[13] Wiklundh and Eriksson, "A Study of the Capacity for Different Element Spacing on Compact MIMO Platforms," in Proceedings of 19th IEEE International Symposium on Personal, Indoor and Mobile Radio Communications, Cannes, 2008.
[14] A. Exton , "QUALITY ISSUES IN MPEG-2 TRANSMISSION APPLICATIONS - BANDWIDTH VERSUS QUALITY," in International Broadcasting Convention, 1997.

[15] Meessen, C. Parisot, Desurmo, and J. Delaigle , "Scene Analysis for Reducing Motion JPEG 2000 Video Surveillance Delivery Bandwidth and Complexity," in IEEE International Conference on Image Processing, Genoa/Italy, 2005

[16] C. T. Lia, "A New Architecture for Transmission of MPEG-4 Video on MPLS Networks," Master's Thesis 87423010,2000

[17] C. A. White, "DELIVERY METHOD FOR INTERNET PROTOCOL TELEVISION (IPTV)," 20100050042, February 25, 2010.

[18] A. Tasleem, "A Simulation Study of Optima Bandwidth Utilization for VBR MPEG-4 Streaming over MPLS-Networks," Lahore University of Management Sciences (LUMS), Lahore , Pakistan, 2004.

[19] L. Li, M. M. Buddhikot, and K. Guo, "Routing Bandwidth Guaranteed Paths with Local Restoration in Label Switched Network," IEEE Journal, vol. 23, no. 2, February 2005

[20] Sulonen, Suvikunnas, Vuokko, J. Kivinen, and Vainikainen, "Comparison of MIMO Antenna Configurations in Picocell and Microcell Environments," IEEE JOURNAL ON SELECTED AREAS IN COMMUNICATIONS, vol. 21, no. 5, pp. 703-712, June 2003.

[21] (2010, April) Radio-Electronics.com. [Online] Available: http://www.radioelectronics.com/info/cellulartelecomms/lte-long-termevolution/3g-lte-basics.php 